\title{
Generation and Near-Field Imaging of Airy Surface Plasmons
}

\author{
Alexander Minovich, ${ }^{1}$ Angela E. Klein, ${ }^{2}$ Norik Janunts, ${ }^{2}$ Thomas Pertsch, ${ }^{2}$ Dragomir N. Neshev, ${ }^{1}$ and Yuri S. Kivshar ${ }^{1}$ \\ ${ }^{1}$ Nonlinear Physics Centre, Centre for Ultrahigh-bandwidth Devices for Optical Systems (CUDOS), \\ Research School of Physics and Engineering, Australian National University, Canberra ACT 0200, Australia \\ ${ }^{2}$ Institute of Applied Physics, Abbe Center of Photonics, Friedrich-Schiller-Universität Jena, 07743 Jena, Germany
}

(Received 12 May 2011; revised manuscript received 5 July 2011; published 6 September 2011)

\begin{abstract}
We demonstrate experimentally the generation and near-field imaging of nondiffracting surface waves, plasmonic Airy beams, propagating on the surface of a gold metal film. The Airy plasmons are excited by an engineered nanoscale phase grating, and demonstrate significant beam bending over their propagation. We show that the observed Airy plasmons exhibit self-healing properties, suggesting novel applications in plasmonic circuitry and surface optical manipulation.
\end{abstract}

DOI: 10.1103/PhysRevLett.107.116802

PACS numbers: 73.20.Mf, 42.25.Fx, 78.67.-n

Airy wave packets constitute a special class of nondiffracting waves that accelerate along parabolic trajectories and exhibit self-healing properties. First suggested more than 30 years ago in the pioneering work by Berry and Balazs [1], they became known to exist in various fields of physics [2]. Despite their well-known nondiffracting properties, Airy beams have been observed only recently in free-space optics $[3,4]$ as one of several types of free-space nondiffracting waves that also include Bessel and Mathieu beams. However, in low-dimensional systems such as graphene [5] and magnetic films [6], the Airy beams are the only beams that do not spread with propagation. As such, Airy beams have recently been suggested theoretically in plasmonics [7] and their manipulation through linear potentials [8] suggests novel opportunities for nondiffractive plasmon optics.

Surface plasmon polaritons (SPPs) are localized or propagating quasiparticles in which photons are coupled to the free electron oscillations in metals. The plasmon field is tightly confined to the metal surface decaying exponentially away from it. Such an effectively planar system with a subwavelength confinement is very attractive for "flatland photonics" including the demonstration of optical nondiffracting SPPs. An analytical solution for the Airy plasmons in the paraxial approximation has recently been suggested [7], showing that Airy plasmons can be indeed possible despite the strong energy dissipation at the metal surface. While nonparaxial effects can play an important role in such purely evanescent Airy waves [9], they will not significantly affect practical applications [7] such as energy routing in plasmonic circuits or surface particle manipulation [10]. As such, the experimental demonstration of plasmonic Airy waves represents an important milestone in the field of plasmonics; however, it still remains an unsolved challenge. Here, we demonstrate the direct generation of nondiffracting Airy surface plasmons and quantitatively characterize their unique features, namely, the propagation along self-accelerating trajectories and self-healing properties.
While mathematically the Airy beams have infinite energy, in practice only truncated Airy beams of finite energy can be realized. In free space, such beams are generated by imprinting a cubic phase onto a Gaussian beam in the Fourier plane, and subsequently transforming it back to the real space by a lens $[3,4]$. However, to excite a surface wave, a wave vector mismatch between an SPP and a freespace wave should be compensated first by utilizing a suitable coupling technique. In our work we show that amplitude-phase modulation required for the excitation of Airy plasmons [7] can be realized simultaneously with the transformation from free-space light into the SPPs. The generation is achieved by a specially designed diffraction pattern and by utilizing the uniqueness of the nondiffracting nature of the Airy plasmons.

In our approach for the experimental generation of Airy plasmons, we use a grating coupling scheme [Fig. 1(a)], with a grating pattern designed to imprint the phase profile of an Airy function, as shown in Figs. 1(b)-1(d). No special measures to modulate the field amplitude have been taken; however, we show that such modulation is not necessary due to the uniqueness of the Airy plasmons as the only nondiffracting beams on the surface. Most importantly, by using this excitation technique we rigorously demonstrate their distinct properties, namely, nondiffracting nature, self-accelerating trajectory [3], and self-healing after passing through surface defects [11]. We note that in the proposed technique the Airy beam is truncated by the size of the grating in the $x$ direction. This truncation does not match the commonly used exponential apodization of the Airy wave [12]; however, the nondiffracting nature of the Airy plasmons assists in the development of the correct field profile. Any additional radiation will quickly diffract due to the small, wavelength-scale size of the grating features used in our experiment. As such, the nondiffracting Airy plasmon will dominate the beam propagation.

In our experiments we generate Airy plasmons on the air-gold interface of a $150 \mathrm{~nm}$ thick gold film deposited on a glass substrate [Fig. 1(a)] by dc sputtering. The 
diffraction pattern is fabricated using a focused ion beam (FIB FEI Helios 600) such that the metal was completely removed from the areas of the rectangular slits. The main lobe of the designed Airy plasmon has a half width of $x_{0}=700 \mathrm{~nm}$. The envelope of the Airy packet represents an oscillating function with alternating positive maxima and negative minima of slowly decaying amplitude. The absolute value of the Airy function therefore represents a sequence of peaks [Fig. 1(b)] that can be observed experimentally in the intensity distribution. The phase distribution of the Airy function thus shows alternating segments with values of 0 and $\pi$ [Fig. 1(c)]. Thus, in the design of the grating, the segments determine the length of the pattern in the transverse direction $x$, and the required phase modulation is achieved by shifting of the next column of slits by half the SPP's wavelength [Fig. 1(d)]. To couple light from free space into the SPP wave at normal incidence, the pattern is periodically repeated along the $z$ direction with a period of $764 \mathrm{~nm}$, equal to the SPP wavelength. The grating is excited from the substrate


FIG. 1 (color online). Generation of Airy plasmons: (a) Schematic of the experimental setup with the engineered grating. The grating is composed of 11 periods of $200 \mathrm{~nm}$ thick slits (in $z$ direction) and varying width (in $x$ direction). The grating is excited from the glass-substrate side with a broad Gaussian beam at $784 \mathrm{~nm}$ and polarization perpendicular to the slits. (b),(c) Absolute value and phase of the amplitude function of the Airy plasmon. The main lobe half width is $x_{0}=700 \mathrm{~nm}$. (d) Grating geometry for generation of Airy plasmons, $\lambda_{\mathrm{SPP}}=$ $764 \mathrm{~nm}$ denotes the SPP wavelengths. side by a $10 \mathrm{~mW} \mathrm{cw}$ diode laser at $784 \mathrm{~nm}$. The beam width onto the grating is $\sim 200 \mu \mathrm{m}$, thus completely covering the grating pattern.

To test if the designed grating indeed couples the freepropagating light into an Airy-type accelerating plasmon, we perform near-field microscopy and image the electric field distribution at the metal surface. The near-field intensity distribution at the gold-air interface is characterized by a near-field scanning optical microscope (NSOM) (Nanonics MultiView 4000). A bent gold-coated fiber tip with aperture size of $150 \mathrm{~nm}$ is used for light collection. The optical signal is then detected by a SPCM-AQR-14 (Perkin-Elmer) single photon counting module. In Fig. 2(a) we superimpose a scanning electron microscope image of the fabricated grating with the measured NSOM image. The near-field distribution clearly shows that at the edge of the grating we generate a surface plasmon that propagates on the metal surface along a curved trajectory for more than $20 \mu \mathrm{m}$. (In fact, two Airy plasmon beams are symmetrically generated on both sides of the grating.) Importantly, the main lobe bends by more than two lobe widths before the wave loses its power due to the plasmon propagation losses. In Fig. 2(b) we compare the experimentally measured near-field profile with the one obtained theoretically by finite difference time-domain (FDTD) calculations and achieve excellent agreement between both. Even though both theory and experiment show some extra light that interferes with the generated Airy plasmon, this additional radiation diffracts quickly and its effect on the Airy plasmon is weak. Numerical tests show that the amount of extra radiation depends most crucially on the size of the first widest slit of the grating and is largely minimized by truncating the slit at 0.2 level of the Airy function [Fig. 1(b)]. The Airy plasmon generated by the diffraction pattern also demonstrates robustness towards excitation wavelength variation. We estimate that the generation bandwidth of the Airy plasmons is as wide as $70 \mathrm{~nm}$. Details on such bandwidth are presented in the Supplemental Material [13].

Importantly, in the experiments in Fig. 2(a) one can also observe the self-healing properties of the Airy plasmon. Namely, that the Airy plasmon beam recovers its shape after passing by a structural defect on the metal surface. Such a defect is seen in Fig. 2(a) at the coordinate $(x, z)=$ $(2.0,12.5) \mu \mathrm{m}$. The measured field distribution shows that this surface defect disturbs the beam profile only locally, as expected for a nondiffracting beam. Further proofs on the strong self-healing properties of the Airy plasmons and quantitative analysis of their healing length are provided by numerical simulations, where we artificially create a hole in the metal film in front of the main Airy lobe. In Figs. 3(a) and 3(b) the Airy plasmon passes through a hole of one or two SPP wavelengths size; however, in both cases it recovers its shape at $4.7 \mu \mathrm{m}$ after the holes as compared to the Airy plasmon on a smooth metal surface [Fig. 2(b)]. 



FIG. 2 (color online). Near-field imaging of Airy plasmons. (a) Superimposed scanning electron microscope image of the grating pattern and the measured near-field intensity profile of the accelerating plasmon. The gold-coated bent fiber probe used for the measurement is most sensitive to the tangential component $\left|E_{\tau}\right|^{2}$ of the electric field. (b) Numerically calculated distribution $\left|E_{\tau}\right|^{2}=\left|E_{z}\right|^{2}+\left|E_{x}\right|^{2}$ at the metal-air interface. The excitation wavelength is $\lambda_{0}=784 \mathrm{~nm}$.

These self-healing properties of the Airy plasmons make them specifically attractive for surface manipulation of nanoparticles, similar to the recent manipulation of microscale objects by free-space propagating Airy beams [14].

An important step in proving the excitation of nondiffracting plasmons modes is to quantitatively characterize the plasmon trajectory. The deflection of the main lobe extracted from the experimental and numerical data as well as the trajectories given by the paraxial approximation [7]
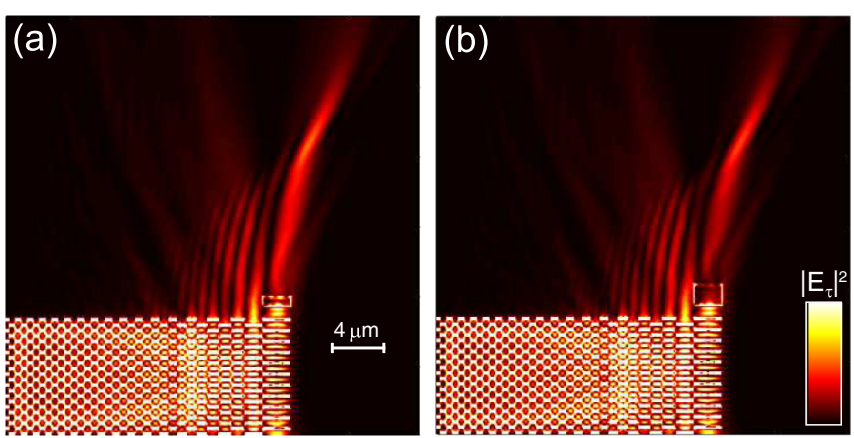

FIG. 3 (color online). Airy plasmon self-healing: Numerically calculated near-field intensity profiles when a hole (shown with a dashed square) is made in the metal film at the path of the main Airy plasmon lobe. (a) The hole length in the $z$ direction is one SPP wavelength, (b) the hole length is two SPP wavelengths. The excitation wavelength is $\lambda_{0}=784 \mathrm{~nm}$. The Airy profile on a smooth metal surface is shown in Fig. 2(b).

and nonparaxial solution calculated according to Ref. [9] (see also the Supplemental Material [13]) are shown in Fig. 4(a). At the initial stage of propagation $(z \sim 3 \mu \mathrm{m})$ we see some stronger variations of the beam deflection [Fig. 4(a)]. However, after this initial stage, the main peak follows a smooth trajectory, which matches well the result of numerical calculations. Our analysis showed that the trajectory can be fitted well to a parabolic function as expected for an Airy beam. Because of the nonparaxiality of the Airy plasmon $\left(\lambda_{0}=784 \mathrm{~nm}, x_{0}=700 \mathrm{~nm}\right)$ both numerical and experimental results deviate from the analytical paraxial solution [7] [Fig. 4(a), solid line]; however, a nonparaxial solution calculated according Ref. [9] predicts the behavior of the Airy plasmon more precise.

The full width at half maximum (FWHM) of the main lobe is a good characteristic for the quality of an Airy plasmon and an additional verification of its nondiffracting nature. It has a constant value in the case of an ideal nondiffracting wave. In Fig. 4(b) we present the FWHM obtained from our experimental and numerical data. In both cases the beam width is affected by the extra radiation at the initial stage of propagation; however, the main-lobe width [Fig. 4(b)] remains practically constant $(\sim 1.5 \mu \mathrm{m})$ over the diffraction-free zone of propagation. This zone is determined by the truncation of the Airy wave packet [12] due to the finite transverse size of our grating. In Fig. 4, the edge of the diffraction-free zone is marked with gray shading at around $15 \mu \mathrm{m}$. Outside this zone the main beam peak starts to quickly expand, losing its Airy profile. Nevertheless, over the diffraction-free propagation we see a significant $(\sim 3 \times)$ beam bending of the Airy plasmon trajectory.

While the measured and simulated FDTD results demonstrate a good agreement, the discrepancies between the nonparaxial solution and the experimental measurements are attributed mainly to the difference in beam apodization. Strictly speaking, the experimental field profile in our case 

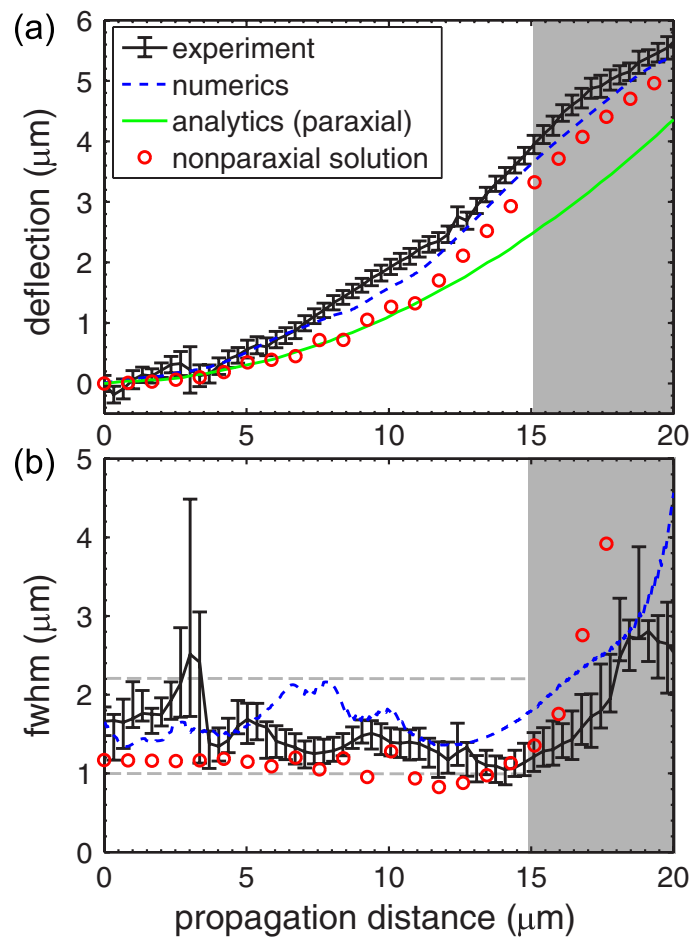

FIG. 4 (color online). Airy plasmon trajectory: (a) Main peak deflection vs propagation distance. (b) Full width at half maximum of the main lobe vs propagation distance. Solid curves with error bars-experimental data; dashed curves - numerical data; solid curves - analytical solution for a paraxial Airy plasmon of $a=0.04$ [7]; circles - nonparaxial solution calculated according to Ref. [9].

is not apodized exponentially as in Ref. [7] and the effective apodization parameter $a$ can only be roughly estimated from the fit of the first highest lobes of the Airy profile. Further improvement of the quality of the generated Airy plasmons can be realized by the adjustment of the slit size in order to modulate the transmission through the grating. While such optimization is in general a complex numerical problem, some basic results of optimization procedure are presented in the Supplemental Material [13].

In conclusion, we have demonstrated the generation of nondiffracting Airy plasmons on a metal surface. We have employed a specially designed diffraction grating that directly couples free-propagating light to plasmonic Airy beams. We have observed experimentally that the Airy plasmons propagate over parabolically curved trajectories and possess unique self-healing properties, being able to quickly recover after passing through obstacles and surface defects. We believe that the demonstration of Airy plasmon beams opens new opportunities for selective on-chip manipulation of nanoparticles [10], optical sensing, photonic circuitry [15], and magnetoelectronics. We anticipate that our results can open further opportunities for generation of subwavelength Airy beams in plasmonics or other surface systems, as recently attempted in spin waves [16]. Finally, novel techniques for nonlinear Airy plasmon generation can be explored, combining the recent nonlinear generation of free-space Airy beams [17] and nonlinear generation of surface plasmons $[18,19]$.

We acknowledge support from the Australian National Computational Infrastructure, the ACT Node of the Australian Nanofabrication Facility, Go8-DAAD Joint Research Cooperation Scheme, the Australian Research Council, the German Research Foundation (DFG), Jena School for Microbial Communication, and the German Federal Ministry of Education and Research (BMBF).

[1] M. V. Berry and N. L. Balazs, Am. J. Phys. 47, 264 (1979).

[2] O. Vallée and M. Soares, Airy Functions and Applications to Physics (Imperial College Press, London, 2010), 2nd ed.

[3] G. A. Siviloglou, J. Broky, A. Dogariu, and D. N. Christodoulides, Phys. Rev. Lett. 99, 213901 (2007).

[4] A. Chong, W. H. Renninger, D. N. Christodoulides, and F. W. Wise, Nat. Photon. 4, 103 (2010).

[5] N.E. Ashkan Vakil, arXiv:1101.3585v1.

[6] V.E. Demidov, S. Urazhdin, and S. O. Demokritov, Nature Mater. 9, 984 (2010).

[7] A. Salandrino and D. N. Christodoulides, Opt. Lett. 35, 2082 (2010).

[8] W. Liu, D.N. Neshev, I. V. Shadrivov, A.E. Miroshnichenko, and Y.S. Kivshar, Opt. Lett. 36, 1164 (2011).

[9] A. V. Novitsky and D. V. Novitsky, Opt. Lett. 34, 3430 (2009).

[10] M. Righini, A. S. Zelenina, C. Girard, and R. Quidant, Nature Phys. 3, 477 (2007).

[11] J. Broky, G. A. Siviloglou, A. Dogariu, and D. N. Christodoulides, Opt. Express 16, 12880 (2008).

[12] G. A. Siviloglou and D. N. Christodoulides, Opt. Lett. 32, 979 (2007).

[13] See Supplemental Material at http://link.aps.org/ supplemental/10.1103/PhysRevLett.107.116802 for theoretical models and numerical calculation details, generation bandwidth, and pattern optimization studies.

[14] J. Baumgartl, M. Mazilu, and K. Dholakia, Nat. Photon. 2, 675 (2008).

[15] T. W. Ebbesen, C. Genet, and S. I. Bozhevolnyi, Phys. Today, No. 5, 61, 44 (2008).

[16] T. Schneider, A. A. Serga, A. V. Chumak, C. W. Sandweg, S. Trudel, S. Wolff, M.P. Kostylev, V.S. Tiberkevich, A. N. Slavin, and B. Hillebrands, Phys. Rev. Lett. 104, 197203 (2010).

[17] T. Ellenbogen, N. Voloch-Bloch, A. Ganany-Padowicz, and A. Arie, Nat. Photon. 3, 395 (2009).

[18] H. J. Simon, D. E. Mitchell, and J. G. Watson, Phys. Rev. Lett. 33, 1531 (1974).

[19] S. Palomba and L. Novotny, Phys. Rev. Lett. 101, 056802 (2008). 